\title{
Diagnosing Latent Tuberculosis Infection in the HIV Era
}

\author{
Philippe H. Lagrange ${ }^{*}, 1$ and Jean Louis Herrmann ${ }^{2}$ \\ ${ }^{1}$ Service de Microbiologie, Hôpital Saint Louis, Paris, France \\ ${ }^{2}$ Service de Microbiologie, Hôpital Raymond Poincaré, Garches, France
}

\begin{abstract}
Tuberculin skin testing (TST) and Interferon-gamma (IFN $\gamma$ ) release assays (IGRAs) are presently the only available assays for the detection of Mycobacterium tuberculosis infected individuals. IGRAs might progressively replace TST, as numerous published reports establish their higher specificity and similar sensitivity when tested in BCG vaccinated, immunocompetent individuals or in populations who may have been in contact with atypical mycobacteria. However, few published reports have commented on their role in TB diagnosis in immunocompromised individuals (HIV, immunosuppressive therapy, cancer...). It is the purpose of this report to review IGRAs published studies in HIV individuals in endemic and non endemic area for tuberculosis (TB). IGRAs were tested in the presence or absence of active TB but correlated to duration of exposure. In newly diagnosed active TB, IGRAs demonstrated a similar sensitivity to TST. In TB non infected individuals, TST and IGRAs also gave similar values when categorization of individuals was correlated to the risk of infection. A higher number of positive IGRAs was observed in individuals from TB endemic areas, in similar proportions to immunocompetent individuals. Comparison between the two IGRAs: QuantiFERON-TB Gold $^{\circledR}$ (QF-TB, Cellestis, Australia) and T-SPOT-TB ${ }^{\circledR}$ (Oxford Immunotec, UK), and against TST, in the same HIV population demonstrates a higher sensitivity of T-SPOT-TB and TST than QF-TB. Indeterminate results, which correspond to the absence of a positive T-cell IFN $\gamma$ response towards phytohemaglutinin (PHA), is a key point when comparing both IGRAs. This PHA control is indicative of the level of immunosuppression observed in the tested individual. QFTB seems to present, in HIV populations, more indeterminate results than T-SPOT-TB. The calibration and/or concentration of PBMC on nitrocellulose membrane for the T-SPOT-TB, as compared to a whole blood assay, might explain this difference, with less indeterminate results with the T-SPOT-TB assay. Neither assay is able to differentiate active TB from latent TB infection (LTBI). Several laboratories have tried new antigenic epitopes to solve this issue. It is of importance that these studies need to be repeated on a larger scale by others to validate their results. Two blood assays might add information characterising the evolution from LTBI to active TB: either by losing protective immunity, as demonstrated by the whole blood killing assay, or by evaluating the kinetics of the antibodies synthesized against M. tuberculosis specific antigens. In conclusion, longitudinal studies are still needed to validate IGRAs and other assays and to define their respective predictive values.
\end{abstract}

Keywords: Tuberculin skin test, Interferon-gamma release assays, HIV-infected, latent tuberculosis infection, active tuberculosis, whole-blood killing assay, ELISA, antibody, PGL-Tb1.

\section{INTRODUCTION}

In most individuals infection with Mycobacterium tuberculosis is contained by the host's immune defences and the infection remains latent $[1,2]$. The World Health Organisation (WHO) has estimated that approximately a third of the world's population is infected with $M$. tuberculosis, the vast majority with Latent Tuberculosis Infection (LTBI) [3]. The M. tuberculosis bacilli that persist in symptom-free immunocompetent individuals with LTBI, can reactivate however, and cause active TB disease in about $10 \%$ of those infected over a lifetime [4]. Currently, it is difficult to predict exactly when and who among the latently infected individuals will develop the disease. Targeted testing and treatment for LTBI in high risk populations is a key component of TB control in many low-incidence, high income countries as a consequence of this risk of progression from LTBI to active TB disease [5].

*Address correspondence to this author at the Service de Microbiologie, Hôpital Saint Louis, Paris, France;

E-mail: philippe-henri.lagrange@sls.aphp.fr
The rational of such preventive treatment is also linked to TB transmission in the community occurring mostly before a diagnosis of TB is made in the index case, even when an optimal TB control program is in place: new and undiagnosed cases are the driving force behind the current TB epidemic [6]. It has been calculated that if each new TB case infected up to 10 susceptible contacts before diagnosis, up to 40 million new infections might occur worldwide every year, adding to the pool of existing LTBI [7]. Implementation of an effective screening program in high risk populations that would identify individuals with LTBI and treat them to prevent disease would be of enormous operational value.

The determinants for increased risk of progression from LTBI to active TB disease are a recent infection with $M$. tuberculosis and several host-related factors, all of which seems to be associated with an impaired cell-mediated immune response [8]. Among this high risk populations, individuals infected with the Human Immunodeficiency Virus (HIV) are the most prone to reactivate the persistent $M$. $t u$ berculosis bacilli. Likewise, after a primary infection, the risk of developing active TB disease in HIV-positive indi- 
viduals is increased many fold, even when antiretroviral chemotherapy is given [9-11], and the incidence of TB is increasing in regions where HIV is prevalent [3]. As described in the Global Plan to Stop TB 2006-2015, management of LTBI in high HIV prevalence settings will be of paramount importance, together with DOTS expansion and provision of a tuberculosis/HIV package of prevention and care, to control and eventually to eliminate tuberculosis [12].

For several decades, identification of individuals with LTBI has been conventionally performed using the Tuberculin Skin Test (TST) [13]. Several studies have demonstrated its prognostic value with correct correlation between a positive test and potential occurrence of active TB [14-16]. Several prospective studies using TST have established the benefit of prophylactic therapy in individuals with LTBI, either in a setting of HIV co-infection or not [17-19]. However, accumulated experience has led to a full understanding of the limitations and drawbacks of TST. The limitations of TST in this setting is the need for two visits [20], the subjective nature of the test placement and its interpretation, and false-positive reactions due to Bacille Calmette-Guérin (BCG) vaccination and exposure to non tuberculous mycobacteria (NTM) [13]. In addition, HIV-infected patients have a higher rate of specific and non-specific unresponsiveness (anergy), particularly with advanced immunosuppression [21-24]. Thus, new tests for diagnosing LTBI are warranted, and ideally such tests should have several characteristics: a high sensitivity in all populations at risk, and a high specificity, regardless of BCG vaccination or infection with environmental NTM. It should also be reliable, stable over time, and with objective criteria for a positive result. Because, most of the LTBI occurred in high incidence, resourcelimited countries; this test should also be inexpensive and easy to perform.

Presently, two in vitro assays called IFN $\gamma$-released assays [IGRAs; QuantiFERON-TB Gold ${ }_{\mathbb{R}}^{\text {in }}$ Tube (QF-TB-IT, Cellestis, Australia) and T-SPOT-TB (Oxford Immunotec, UK)] have been developed and are commercially available. They measure the T-cell IFN $\gamma$ production after 16 to $24 \mathrm{~h}$ of contact with $M$. tuberculosis specific antigens coded by the region of deletion 1 or RD1 (ESAT-6 + CFP-10 for T-SPOTTB, and ESAT-6 + CFP-10 + Tb7.7 for QF-TB-IT). Both assays have a negative and a positive control, the latter being represented by the T-cell response towards the phytohemaglutinin PHA. Both assays, in addition to TST, are the only available assays for the diagnosis of M. tuberculosis infected individuals.

Comparison between IGRAs and TST has been performed, either in well defined active TB patients to establish their respective sensitivity or in well defined controls without any contact risk with a TB index case to establish their respective specificity. As is clearly evident, we do not benefit from a gold standard to define such a control population to compare with active TB patients where a positive culture represents the gold standard.

To deal with LTBI diagnosis, investigators have used two approaches. In the first one, investigators compared directly the results of the TST with those of the IGRAs and calculated a degree of agreement (using the Cohen's kappa coefficient). With the second approach, investigators designed studies to establish the extent of which the test performance fits with a defined attribute (e.g., the likelihood of infection based on clinical or epidemiological characteristics). This second approach is consistent with a causal relationship and thereby strengthens the assessment of the validity (e.g., the gradient of exposure as an indicator of the likelihood of recent LTBI).

Several recent reviews have shown considerable promise for IGRA as a new diagnostic tool for LTBI in immunocompetent TB infected individuals [25-30]. IGRAs when compared to TST do not cross-react with immune responses elicited by BCG vaccination and NTM exposure. In addition, IGRA results have shown a higher sensitivity than TST in low endemic TB countries. Recent meta-analysis has further emphasized these results with suboptimal sensitivities (TST: 71\%; QF-TB: 76\%; ELISPOT: 88\%) and high pooled specificities (TST: 66\%; QF-TB: 97\%, ELISPOT: 92\%) [31]. Both assays were more specific than TST in samples from individuals vaccinated with BCG. Discordant TST and IGRAs reactions were frequent and largely unexplained (definitions used for the positive test results, the study population, the sample sizes, and the study design) [32-34].

Compared to the extensive numbers of studies performed in immunocompetent individuals infected with M. tuberculosis, there are only limited data describing IGRA performance in HIV-infected individuals, who's immunological impairment may affect the performance of these lymphocyte-based assays. It is the purpose of this review to analyze the published studies relative to the usefulness and limitations of the IGRA in HIV infected individuals in comparison with the TST.

\section{REVIEW}

\section{New T-Lymphocyte-Based Tests for LTBI}

\subsection{The IGRA Performances in HIV Infected Individuals}

The first study was done in Zambian HIV-positive [35]. An "in-house" PPD and RD1 antigens ELISPOT was compared to TST in 39 HIV-TB coinfected patients (with advanced immunosuppression), $11 \mathrm{HIV}$-negative patients with active TB disease and in the 54 HIV-negative asymptomatic Zambian healthy adults. PPD and RD1 ELISPOT gave identical results in HIV uninfected patients $(100 \%$, Table 1); but gave fewer positive responses in HIV-positive co-infected TB patients. RD1 ELISPOT positive responses were, however, more frequent than PPD ELISPOT positive responses in the same HIV-positive co-infected TB patients (Table 1). Finally, TST and PPD ELISPOT were more often positive than RD1 ELISPOT in healthy control individuals (Table 1) [35]. Of note, three RD1 ELISPOT positive, HIV-positive but TB negative were PPD ELISPOT and TST negative [35]. IGRAs might perform well in HIV-infected patients even better as compared to TST. However, this "in-house" assay was performed in a relatively low number of patients and the $\mathrm{CD} 4+$ cell counts per patient were not reported.

Following this study, several recent reports evaluated IGRAs performance in HIV- infected patients with active TB (Table 2) [36-38]. The prevalence of the TST positive responses was always lower than the proportion of IGRA positive responses in HIV-infected patients with active TB. In this group of patients, the performances of the T-SPOT and 
Table 1. Frequency of Positive Results in Zambian TB Patients and Healthy Individuals According to their HIV Status Tested with TST or with ELISPOT. Data from Chapman et al. [35]

\begin{tabular}{|c|c|c|c|c|}
\hline \multirow{2}{*}{ Patients Tested } & \multirow{2}{*}{ HIV Status } & \multirow{2}{*}{ TST (5 TU) } & \multicolumn{2}{|c|}{ ELISPOT } \\
\hline & & & PPD & ESAT6/CFP10 \\
\hline TB disease & $\begin{array}{l}\text { negative } \\
\text { positive }\end{array}$ & $\begin{array}{l}\mathrm{NT}^{*} \\
\mathrm{NT}^{*}\end{array}$ & $\begin{array}{c}11 / 11(100 \%) \\
28 / 39(72 \%)\end{array}$ & $\begin{array}{c}11 / 11(100 \%) \\
35 / 39(90 \%)\end{array}$ \\
\hline Healthy Zambian & $\begin{array}{l}\text { negative } \\
\text { positive }\end{array}$ & $\begin{array}{c}28 / 35(80 \%) \\
5 / 14(36 \%)\end{array}$ & $\begin{array}{c}45 / 54(83 \%) \\
6 / 21(29 \%)\end{array}$ & $\begin{array}{c}37 / 54(69 \%) \\
9 / 21(43 \%)\end{array}$ \\
\hline Healthy UK** & Negative & NT* & $33 / 40(83 \%)$ & $0 / 40(0 \%)$ \\
\hline
\end{tabular}

*NT: non tested

**30/48 (80\%) have been BCG vaccinated.

QF-TB assays were identical showing 85 to $90 \%$ of positive responses.

In HIV-infected patients without any TB disease, IGRAs results were more variable and might reflect the different TB epidemiological situations in the countries where the studies were performed. Higher positive responses were observed in patients from South Africa compared to European patients.

Twice the number of published studies described specific results that were obtained in individuals with LTBI comparing the respective prevalence of positive results in TST with those with IGRAs, half being evaluated in low endemic regions, the other half in high endemic regions (Table 3) [3944].

In three large cohorts performed in low endemic countries, data showed that only a limited number of HIVinfected individuals had positive IGRA (4.1 to $8.5 \%$ ), and almost $80 \%$ of these individuals with IGRA positive results had risk factors for LTBI (history of exposure, long term residency in a high endemic country) [39-41]. When tested simultaneously, TST and QF-TB-IT gave comparable positive results.

In the three studies performed in high endemic countries, a higher frequency of IGRA and TST positive results was observed related to higher transmission rate of $M$. tuberculosis in this population [42-44]. TST positive results ranged from $83 \%$ (in HIV-negative LTBI individuals) to $21.4 \%$
(HIV-positive LTBI individuals). In the study performed by Rangaka and coworkers, significantly fewer $(p<0.01)$ TST positive results were obtained in the HIV-infected group as compared to the HIV-uninfected group regardless of TST cutoff [42]. A similar decrease in positive TST results in HIV-infected individuals with LTBI was shown recently in India [45]. In comparison, IGRA results gave more homogeneous results. When the 2 IGRAs were tested simultaneously, higher numbers of individuals scored with a T-SPOT positive response as compared to QF-TB-G, in HIV-infected (52\% vs 43\%) and HIV-non infected individuals (59\% vs $46 \%$, respectively). However, such differences were not statistically significant $(\mathrm{p}=0.07)$ [42].

\subsection{Stratification of Results in HIV-Infected Individuals by CD4+ Cell Count}

Both IGRAs depend predominantly on the CD4+-T-cells recognition of $M$. tuberculosis antigens. Test performance in the HIV-infected group will be affected by the absolute CD4+-T-cells count. Similarly, the positive control (using non-specific stimulation with PHA) depends on absolute CD4+ cell count. The PHA control is indicative of the level of immunosuppression observed in the tested individual, and interpretation of IGRAs depends on its positive result. A negative PHA result will confer an indeterminate response of the IGRAs. These two aspects have been explored by stratifying the CD4+ T-cells count with IGRAs results [39-44].

Table 2. Proportion (in Percentage) of IGRA Indeterminate and Positive Results in Groups of HIV-Infected Patients with Active Tuberculosis and in HIV-Positive Patients without Tuberculosis Tested with TST, T-SPOT-TB and QuantiFERON -TB

\begin{tabular}{|c|c|c|c|c|c|c|c|}
\hline \multirow{3}{*}{ Reference } & \multirow{3}{*}{ Diagnosis } & \multirow{3}{*}{ Number Subjects } & \multirow{3}{*}{ Mean CD4 $+/ \mu \mathrm{L}$} & \multicolumn{4}{|c|}{ Tests } \\
\hline & & & & \multicolumn{4}{|c|}{ IGRA } \\
\hline & & & & TST & SPOT & QF-TB & Indeterminate \\
\hline \multirow{2}{*}{ Vincenti (Italy) [36] } & Active TB & 45 & 152 & 47.0 & 84.6 & 84.6 & 20.0 \\
\hline & Non TB & 66 & 236 & 39.9 & 35.7 & 25.0 & 18.2 \\
\hline \multirow{3}{*}{ Rangaka (South Africa) [37] } & Active TB & 41 & 167 & 67.0 & 90.0 & 90.0 & 12.2 \\
\hline & Non TB & 41 & 464 & 51.0 & 75.0 & 79.0 & $7 ; 3$ \\
\hline & HIV (-) controls & 41 & $\mathrm{NA}^{*}$ & 69.0 & 83.0 & 83.0 & 0.0 \\
\hline \multirow{2}{*}{ Clark (UK) [38] } & Active TB & 154 & 209 & $\mathrm{NT}^{* *}$ & 90.3 & $\mathrm{NT}^{* *}$ & 4.5 \\
\hline & LTBI & 47 & 294 & $\mathrm{NT}^{* *}$ & 20.0 & $\mathrm{NT}^{* *}$ & 4.5 \\
\hline
\end{tabular}

\footnotetext{
* NA: non applicable.
}

** NT: non tested. 
Table 3. Proportion (in Percentage) of IGRA Indeterminate and Positive Results in Cohorts of HIV-Infected Patients with Latent Tuberculosis Infection and in HIV-Negative Control Patients Tested with TST, T-SPOT-TB and/or QuantiFERON -TB in Low or High Endemic Tuberculosis Areas

\begin{tabular}{|c|c|c|c|c|c|c|c|}
\hline \multirow{2}{*}{ Reference } & \multirow{2}{*}{ Subjects } & \multirow{2}{*}{ Number } & \multirow{2}{*}{ Mean CD4+/ $\mu \mathrm{L}$} & \multirow{2}{*}{ TST (\%) } & \multicolumn{3}{|c|}{ IGRA Results } \\
\hline & & & & & T-SPOT & QF-TB & Indeterminate \\
\hline Brock (Denmark) [39] & cohort & 590 & 523 & NT & NT(a) & 4.1 & $3.4^{*}$ \\
\hline Luetkemeyer (USA) [40] & cohort & 294 & 132 & 9.3 & $\mathrm{NT}(\mathrm{a})$ & $8.5^{*}$ & $5.1^{*}$ \\
\hline Jones (USA) [41] & cohort & 201 & 453 & 6.8 & NT & 5.8 & $4.9^{*}$ \\
\hline \multirow{2}{*}{ Rangaka (South Africa) [42] } & $\mathrm{HIV}(+)$ & 74 & 392 & $52 *$ & 52.0 & 43.0 & $7.0^{*}$ \\
\hline & HIV(-) & 86 & $\mathrm{NA}(\mathrm{b})$ & 83 & 59.0 & 46.0 & 2.0 \\
\hline Karam (Senegal) [43] & cohort & 285 & 180 & $21.4^{*}$ & $50.6^{*}$ & NT & $13.3^{*}$ \\
\hline Lawn (South Africa) [44] & cohort & 40 & 114 & 43 & 62.0 & NT & 10.0 \\
\hline
\end{tabular}

(a); NT: non tested; (b); NA: non applicable.

* CD4+ cell counts dependent frequency.

Variable percentages of indeterminate results were noticed according to the CD4+ cell count using QF-TB-IT [3941]; with a higher proportion of indeterminate results in patients with low CD4+ cell counts. In individuals with CD4+ cell count lower than 100 cells $/ \mu \mathrm{L}$ proportion of indeterminate results were $24 \%, 16 \%$, and $37 \%$ in these three studies, respectively (Fig. 1) [39-41]. In studies using the T-SPOT$\mathrm{TB}$, fewer indeterminate results were observed. Numbers of interpretable T-SPOT-TB assays seemed less influenced by the CD4+ cell counts (Fig. 2), as already described by Dedha et al. [46].

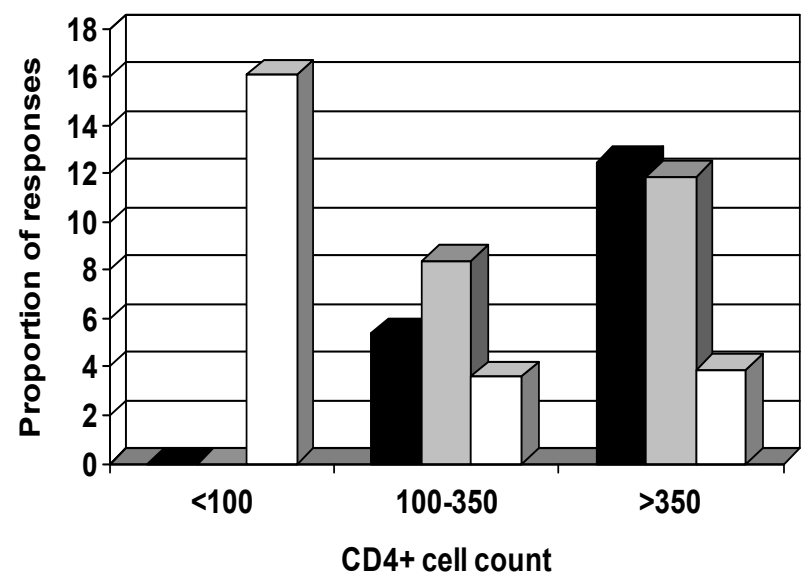

Fig. (1). Percentage of indeterminate responses (white column) positive responders to QuantiFERON-TB-IT (black column) and to TST (grey column) stratified by CD4+ cell count. Data from Jones et al. [41].

For this reason, enhanced performances in a higher proportion of tuberculosis identified infected subjects in the lower CD4+ cell count group was observed for T-SPOT-TB as compared to QF-TB-G or QF-TB-IT (Figs. 1,2).

In conclusion, commercially available IGRAs have evolved rapidly over the past decade. The latest generation of QF-TB-IT uses more specific antigens and is simpler to perform. In our review, the different studies performed in HIV-positive patients showed that, in contrast to the TST, HIV infection does not appear to substantially undermine the IGRA responses in patients with active TB [36-38] or LTBI
[39-44]. However, the proportion of both QF-TB and TSPOT-TB positive results decreases with advanced immune suppression; such a decline seems to be more important for QF-TB than for the ELISPOT assay. Moreover, the proportion of indeterminate results increases with advanced immune suppression and again is more likely with QF-TB-IT than with T-SPOT-TB. Because lymphopenia is often found in advanced HIV-associated immunodeficiency [47], a normalized input of PBMC used in ELISPOT assays may partly explain why this assay appears to retain its efficiency among patients with low $\mathrm{CD} 4+$ cell counts. The other commercially available IGRAs (QF-TB-G, or QF-TB-IT), although producing easier logistic calculation in which the cell input is not normalized, could suffer from a high rate of indeterminate results among those HIV-infected patients with advanced immunodeficiency [39-41].

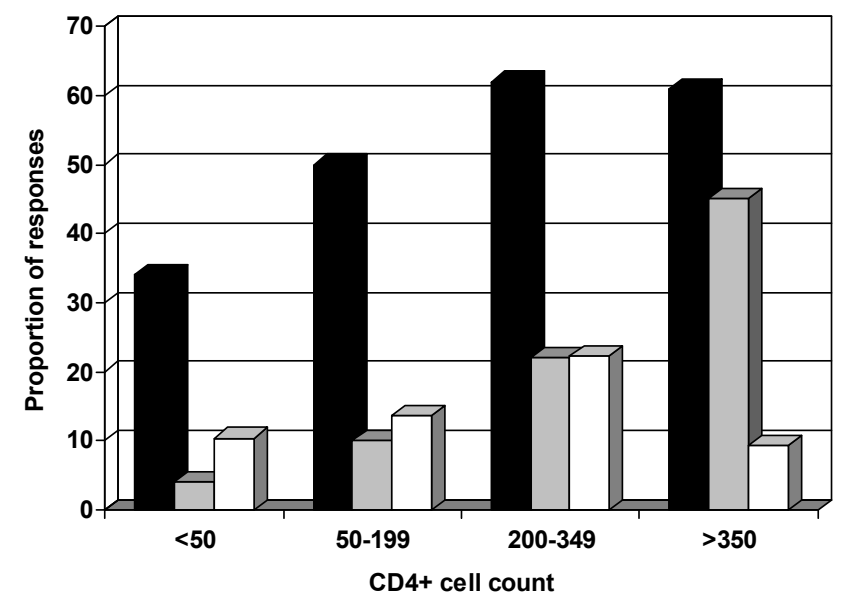

Fig. (2). Percentage of indeterminate responses (white column) positive responders to ESAT-6/CFP10 ELISPOT (black column) and to TST (grey column) stratified by CD4+ cell count. Data from Karam et al. [43].

\subsection{Need for Further Studies}

The two major limitations of these new ex vivo IGRAs for diagnosing LTBI are firstly, that they are not able to differentiate LTBI from active TB and secondly, that they are not predictive of the development of active TB. 
Recently Rangaka and coworkers [37, 42] reported an interesting approach to better identify patients with active TB among HIV-infected patients. This method directly correlates the ELISPOT results of RD1 proteins stimulation with the CD4+ T-cell count of each single patient. Using the ratio of combined T-SPOT-TB results to CD4+ cell count the authors showed that a ratio $>1$ was significantly more frequently observed in patients with active TB compared to latent TB. However, recent published data seemed to contradict such a prediction showing that the indicated ratio was not able to distinguish LTBI from active TB [48].

To be able to identify those individuals who do not progress to active disease, large scale-scale cohort studies with long-term follow-up of untreated populations exhibiting positive results at baseline are required. Ongoing studies are in progress in endemic area trying to evaluate the risk for active disease associated with a particular test measure (quantitative versus dichtotomic results), as shown in uninfected HIV individuals [49, 50].

\subsection{New Directions for New Tests}

Several research groups have evaluated other RD1 or RD coded protein or peptides than those developed by the commercial companies. Different selected RD1 peptides have been developed and tested in Italy by Goletti and coworkers. The authors showed that an assay based on RD1 selected peptides has a higher diagnostic accuracy for active TB in a clinical setting compared with commercially available assays based on RD1 overlapping peptides in HIV-infected or uninfected patients [36, 51, 52].

Other developments concern antigens present at the surface of M. tuberculosis. One group has assessed the diagnostic potential of a novel $28-\mathrm{kD}$ mycobacterial protein, the heparin-binding hemaglutinin (HBHA), previously shown to stimulate high levels of IFN- $\gamma$ secretion by the peripheral blood lymphocytes of LTBI subjects in comparison with QFTB-IT [53]. The conclusion of the authors was that the commercially available IGRA may underestimate the incidence of LTBI, whereas the use of HBHA may combine the operational advantages of IGRAs with higher sensitivity and specificity for latent TB infection [53].

Other groups have tried to measure the different cytokines (IFN- $\gamma$, IL-2, IL-10) released after stimulation with selected antigens to differentiate a population at high risk of active TB, showing a higher ratio of IFN- $\gamma / \mathrm{IL}-10$ in protected individuals [54].

\section{New Ex Vivo Cell-Mediated Killing Assay}

Other researchers are looking for biomarkers for innate immunity or acquired immune protection. It has been reported that in vitro cell-mediated killing assays may provide a promising measure of the functional capacity of cells to kill mycobacteria [55].

Whole blood killing assays (WBKA) have the advantage of including all cells with potential anti-mycobacterial activity compared to infection of isolated differentiated monocytes and are more easily adapted for use in large scale studies. Inhibition of in vitro growth of the bacilli has been measured using different techniques: conventional liquid medium culture (BACTEC system, BD), or luminescent readout of reporter-gene-tagged BCG (BCG lux) expressing a recombinant luciferase enzyme.

Using the second methodology, a study reported an association between low $\mathrm{CD} 4+$ cell counts, low IFN- $\gamma$ production and impaired ability to regulate growth of $M$. bovis BCG in blood from HIV-infected children in South Africa [56]. A more recent study from the same authors reported that this WBKA was able to monitor in vitro the anti-mycobacterial immune response of HIV-infected children during antiretroviral therapy [57]. The authors confirmed their earlier results showing that before HAART the blood from HIVinfected children demonstrated a low ability to restrict the growth of BCG in the functional WBKA; in contrast the introduction of HAART was followed by rapid and sustained reconstitution of specific antimycobacterial immune response, measured as a decrease in growth of the bacilli. This in vitro model mirrors the in vivo observation of decrease susceptibility to TB in HIV-infected adults receiving HAART [58].

\section{Preclinical Detection of Antibody}

Tests for serodiagnosis of TB, either based on ELISA or immunochromatographic strip technology, have been developed and have been evaluated in immunocompetent patients with active TB. The tests, with the best performance characteristics, have an observed sensitivity and specificity around $60 \%$ and $90 \%$, respectively [59], demonstrating that the existing serological tests cannot be used as stand-alone diagnostic test for TB. Moreover, it has been generally observed that HIV-infected individuals with TB have suppressed antibody levels [60]. In this last study, the authors showed that while all HIV-negative and PPD-positive patients had IgG antibodies recognizing the 38-, 28-, and 19-kDa M. tuberculosis antigens, only $26 \%$ of those HIV-positive and PPDpositive (all with $<400 \mathrm{CD} 4+$ cells $/ \mathrm{mm} 3$ ) and none of the HIV-positive tuberculosis patients recognized these antigens. In an effort to increase the performance of potential serological tests, it was relevant to explore whether novel proteins or non-protein antigens specific for $M$. tuberculosis were able to significantly increase sensitivity and specificity. Several proteins, TB16.3, TB9.7, U1, and Mtb81, have been described as serodiagnostic antigens that perform as well as or even better in the HIV-infected group, as recently reviewed $[61,62]$.

Different authors have also shown that antibodies detecting glycolipid antigens (PGL-TB1, DAT), specific for $M$. tuberculosis, were present in HIV-TB co-infected patients with levels higher than those in HIV uninfected TB patients $[63,64]$. Moreover, antibodies to two recombinant proteins (MPT51 and Mtb81), and PGLTb1 or DAT antigens have been shown to be present in serum samples obtained during sub-clinical TB in a high proportion of HIV-infected patients tested [63-65].

Although, ex vivo tests based on cellular immune response (IGRAs) performed in HIV-infected individuals with advanced immunodeficiency (with CD4+ lower than 100 cells $/ \mu \mathrm{L}$ ) have been shown to be associated with a high proportion of indeterminate results, the anti-PGL-Tb1 antibodies levels have been demonstrated to be independent of the CD4+ cell counts [63]. Thus, insight into the serological responses based on anti-glycolipid antigens elicited during 
sub-clinical TB could provide additional markers that identify incipient infection with $M$. tuberculosis before progression to clinical disease occurs $[63,65]$.

\section{Potential New Algorithm for Detecting and Treating HIV-Infected Persons at High Risk for Progression to Active TB}

Using the various blood assays described above, and depending on their validation in large scale longitudinal studies, a new algorithm might be considered (Fig. 3).

In low or high endemic TB areas, all HIV-infected individuals should be first tested with IGRAs (QF-TB-IT or TSPOT-TB) and then stratified according to their level of immuno- suppression. Those individuals with a CD4+ cell count higher than $50-100$ cells/ $\mu \mathrm{L}$ will potentially have either a positive or a negative IGRA response. Individuals with a negative response (meaning absence of recent TB transmission), should have serial IGRA testing at regular interval (every 6 months). Individuals with a positive response (meaning the presence of recent TB transmission) should be tested with the WBKA to evaluate their potential antimycobacterial immune protection. If a positive response (surrogate maker showing the capacity to restrict in vitro growth of M. tuberculosis or BCG) is detected, these individuals could be considered as immune and should not receive any anti tuberculous prophylaxis. If a negative WBKA response is detected, such recently infected individuals without any specific immunity should be considered for anti TB chemoprophylaxis. A certain proportion of Individuals, with $<50-100$ $\mathrm{CD} 4+$ cell count $/ \mu \mathrm{L}$ may produce an IGRA positive response and the same protocol as above should be followed for IGRA positive response. For those individuals with indeterminate or negative results with IGRA, the only suitable test that might demonstrate infection with TB is the measurement of circulating IgG anti-PGL-Tb1 antibody. Individuals with ELISA positive results should be considered at high risk and should received anti-TB chemoprophylaxis after eliminating active TB disease. A systematic approach for blood culture testing to detect circulating mycobacteria should also be considered. For those without circulating antiPGL-Tb1 antibody should be considered for empirical HAART [66].

\section{CONCLUSIONS}

There is a definite need to diagnose and treat LTBI in all $\mathrm{HIV}$-infected individuals at high risk of progression to active TB disease. These individuals should receive anti-TB chemoprophylaxis after thorough testing to eliminate active TB disease. IGRAs based on specific RD1 peptides have shown promising results for diagnosing LTBI not only in immunocompetent individuals, but also in HIV-infected persons at higher risk for development of active disease. Although, these dichotomous (positive/negative) measures cannot distinguish between LTBI and active disease, operational research clinical studies are ongoing to demonstrate their potential role in predicting the risk of progression. Evidence coming from studies showing a high ratio of combined quantitative T-SPOT-TB results to CD4+ cell count in patients with active TB should be confirmed in different settings in order to classify patients with active TB from those with LTBI. It is thus likely that tuberculin skin testing being largely inadequate in these immunocompromised patients will be in the next future substituted for the IGRAs.

Other issues need to be resolved in relation to the identification of correlates of immune protection or "functional biomarkers" that will facilitate the rational design for drugs

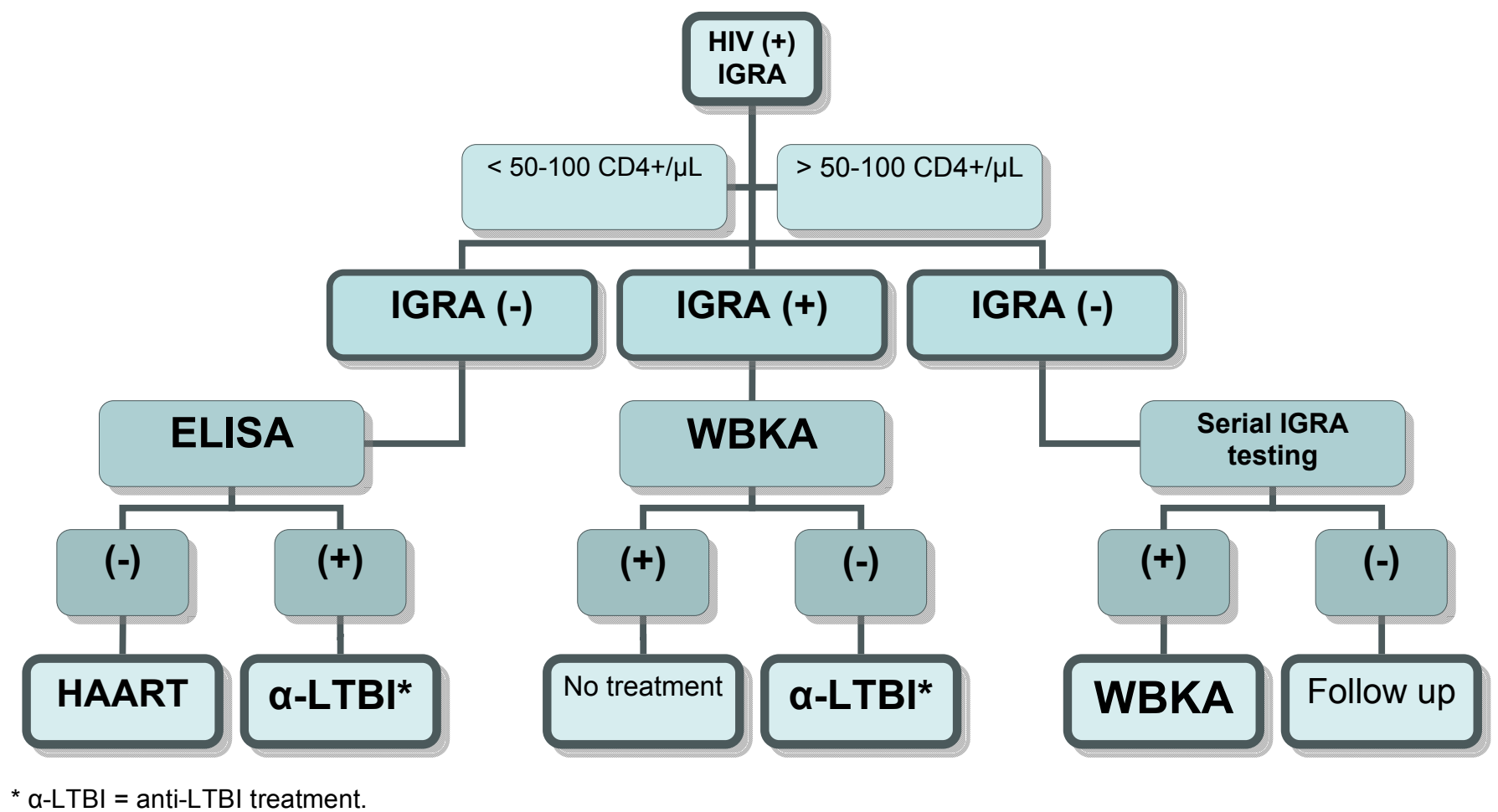

Fig. (3). New possible algorithm for targeting HIV-infected individual establishing the highest risk group and the specific treatment chemoprophylaxis. IGRA (Interferon-gamma release assay), WBKA (Whole Blood Killing Assay), ELISA (antibody assay with PGL-Tb1). 
for the clearance of TB infection. After validation, the WBKA will find its right place among a new algorithm that might facilitate the management of HIV-infected individuals at high risk of progression. Similarly, antibody markers might predict ongoing progression from latent to active TB in HIV-positive subjects, including those with negative PPD skin test or with indeterminate IGRA results.

\section{ACKNOWLEDGEMENTS}

We greatly acknowledge Dr. Ben Marshall (Southampton University Hospitals Trust, UK) for careful review of the manuscript.

\section{REFERENCES}

[1] Jasmer RM, Nahid P, Hopewell PC. Clinical practice. Latent Tuberculosis infection. N Engl J Med 2002; 347: 1860-1866.

[2] Tufariello JM, Chan J, Flynn JL. Latent tuberculosis: mechanisms of the host and bacillus that contribute to persistent infection. Lancet Infect Dis 2003; 3: 578-590.

[3] World Health Organisation. Global Tuberculosis ControlSurveillance, Planning, Financing. WHO report 2005, Geneva, Switzerland;2005.

[4] Styblo K. Recent advances in epidemiological research in tuberculosis. Adv Tuberc Res 1980; 20: 1-63.

[5] American Thoracic Society. Targeted tuberculin testing and treatment of latent tuberculosis infection. Am J Respir Crit Care Med 2000; 161: S221-247.

[6] Chin DP, Crane CM, Diul MY, et al. Spread of Mycobacterium tuberculosis in a community implementing recommended elements of tuberculosis control. JAMA 2000; 283: 2968-2974.

[7] Whalen CC. Diagnosis of Latent Tuberculosis Infection. JAMA 2004; 293: 2785-2787.

[8] Horsburgh CR jr. Priorities for the treatment of latent tuberculosis infection in the United States. N Engl J Med 2004; 350: 2060-2067.

[9] Badri M, Wilson D, Wood R. Effect of highly active antiretroviral therapy on incidence of tuberculosis in South Africa: a cohort study. Lancet 2002; 359: 2059-2064.

[10] Lawn SD, Bekker LG, Wood R. How effectively does HAART restore immune responses to Mycobacterium tuberculosis? Implications for tuberculosis control. AIDS 2005; 19: 1113-1124.

[11] Seyler C, Toure S, Messou E, Bonard D, Gabillard D, Anglaret X. Risk factors for active tuberculosis after antiretroviral treatment initiation in Abidjan. Am J Respir Crit Care Med 2005; 172: 123-127.

[12] Stop TB Partnership and WHO. The Global Plan to Stop TB 20062015. Geneva: World Health Organisation, 2006. http://www. stoptb.org/globalplan/plan main.asp (accessed Jan 05, 2008).

[13] Huebner RE, Schein MF, Bass JB Jr. The Tuberculin Skin Test. Clin Infect Dis 1993; 17: 968-975.

[14] Comstock GW, Livesay VT, Wollpert SF. The prognosis of a positive tuberculin reaction in childhood and adolescence. Am J Epidemiol 1974; 99: 131-138.

[15] Watkins RE, Brennan R, Plant AJ. Tuberculin reactivity and the risk of tuberculosis: a review. Int J Tuberc Lung Dis 2000; 4: 895903.

[16] Tuberculosis Research Centre (ICMR), Chennai, India. Association of initial tuberculin sensitivity, age and sex with the incidence of tuberculosis in South India: a 15 year follow-up. Int J Tuberc Lung Dis 2003; 7: 1083-1091.

[17] Ferebee SH. Controlled chemoprophylaxis trials in tuberculosis. A general review. Bull Tuberc 1970; 26: 28-106.

[18] Whalen CC, Johnson JL, Okwera A, et al. A trial of three regimens to prevent tuberculosis in Ugandan adults infected with the human immunodeficiency virus. Uganda-Case Western Reserve University Research Collaboration. N Engl J Med 1997; 337: 801-808.

[19] Nuwaha F. Chemoprophylaxis for tuberculosis in HIV-infected individuals in sub-Saharan Africa. East Afr Med J 1998; 75: 520527.

[20] Malotte CK, Rhodes F, Mais KE. Tuberculosis screening and compliance with return for skin test reading among active drug users. Am J Public Health 1998; 88: 792-796.

[21] Chin DP, Osmond D, Page-Shafer K, et al. Reliability of anergy skin testing in persons with HIV infection. The pulmonary Compli- cations of HIV Infection Study Group. Am J Respir Crit Care Med 1996; 153: 1982-1984.

[22] Caiaffa WT, Graham NM, Galai N, Rizzo RT, Nelson KE, Vlahov D. Instability of delayed-type hypersensitivity skin test anergy in human immunodeficiency virus infection. Arch Intern Med 1995; 155: 2111-2117.

[23] Duncan LE, Elliott AM, Hayes RJ, et al. Tuberculin sensitivity and HIV-1 status of patients attending a sexually transmitted diseases clinic in Lusaka, Zambia: a cross-sectional study. Trans R Soc Trop Med Hyg 1995; 89: 37-40.

[24] Markowitz N, Hansen NI, Wilcosky TC, et al. Tuberculin and anergy testing in HIV-seropositive and HIV-seronegative persons. Pulmonary Complications of HIV Infection Study Group. Ann Intern Med 1993; 119: 185-193.

[25] Pai M, Riley LW, Colford JM Jr. Interferon- $\gamma$ assays in the immunodiagnosis of tuberculosis: a systematic review. Lancet Inf Dis 2004; 4: 761-776.

[26] Andersen P, Munk ME, Pollock JM, Doherty TM. Specific immune-based diagnosis of tuberculosis. Lancet 2000; 356: 10991104.

[27] Dheda K, Udwadia ZF, Hugget JF, Johnson MA, Rook GA. Utility of the antigen-specific interferon-gamma assay for the management of tuberculosis. Curr Opin Pulm Med 2005; 11: 195-202.

[28] Rothel JS, Andersen P. Diagnosis of latent Mycobacterium tuberculosis infection: is the demise of the Mantoux test imminent? Expert Rev Anti Infect Ther 2005; 3: 981-993.

[29] Richeldi L. An update on the diagnosis of tuberculosis infection. Am J Respir Crit Care Med 2006; 174: 736-742.

[30] Lagrange PH, Simonney N, Herrmann JL. New immunological tests in the diagnosis of tuberculosis. Rev Mal Respir 2007; 24: 453-472.

[31] Menzies D, Pai M, Comstock G. Meta-analysis: new tests for diagnosis of latent tuberculosis infection: areas of uncertainty and recommendations for research. Ann Intern Med 2007; 146: 340-354.

[32] Lee JY, Choi HJ, Park IN, et al. Comparison of two commercial interferon-gamma assays for diagnosing Mycobacterium tuberculosis infection. Eur Respir J 2006; 28: 24-30.

[33] Kang YA, Lee HW, Yoon HI, et al. Discrepancy between the tuberculin skin test and the whole-blood interferon-gamma assay for the diagnosis of latent tuberculosis infection in an intermediate tuberculosis burden country. JAMA 2005; 293: 2756-2761.

[34] Pai M, Kalantri S, Dheda K. New tools and emerging techniques for the diagnosis of tuberculosis: part I. Latent tuberculosis. Expert Rev Mol Diagn 2006; 6: 413-422.

[35] Chapman ALN, Munkanta M, Wilkinson KA, et al. Rapid detection of active and latent tuberculosis infection in HIV-positive individuals by enumeration of Mycobacterium tuberculosis specific T cells. AIDS 2002; 16: 2285-2293.

[36] Vincenti D, Carrara S, Butera FO, et al. Response to region of difference 1 (RD1) epitopes in human immunodeficiency virus (HIV)-infected individuals enrolled with suspected active tuberculosis: a pilot study. Clin Exp Immunol 2007; 150: 91-98.

[37] Rangaka MX, Diwakar L, Seldon R, et al. Clinical, immunological and epidemiological importance of antituberculous $\mathrm{T}$ cell responses in HIV-infected Africans. Clin Infect Dis 2007; 44: 1639-1646.

[38] Clark SA, Martin SL, Pozniak A, et al. Tuberculosis antigenspecific immune responses can be detected using enzyme-linked immunospot technology in human immunodeficiency virus (HIV)1 patient with advanced diseases. Clin Exp Immunol 2007; 150: 238-244.

[39] Brock I, Ruhwald M, Lundgren B, Westh H, Mathiensen LR, Ravn P. Latent tuberculosis infection in HIV positive, diagnosed by the M. tuberculosis specific Interferon- $\gamma$ test. Respir Res 2006; 7: 56 (Published online 2006 April 1. doi: 10.1186/1465-9921-7-56).

[40] Luetkemeyer AF, Charlebois ED, Flores LL, et al. Comparison of an Interferon- $\gamma$ release assay with tuberculin skin testing in HIVinfected individuals. Am J Respir Crit Care Med 2007; 175: 737742.

[41] Jones S, de Gijsel D, Wallach FR, Gurtman AC, Shi Q, Sacks H. Utility of QuantiFERON-TB Gold in-tube testing for Latent TB infection in HIV-infected individuals. Int J Tuberc Lung Dis 2007; 11: 1190-1195.

[42] Rangaka MX, Wilkinson KA, Seldon R, et al. Effect of HIV-1 infection on T-cell-based and skin test detection in tuberculosis infection. Am J Respir Crit Care Med 2007; 175: 514-520. 
[43] Karam F, Mbow F, Fletcher H, et al. Sensitivity of IFN- $\gamma$ release assay to detect latent tuberculosis infection is retained in HIVinfected patients but dependent on HIV/AIDS progression. PLoS ONE 2008; 3 (1): e 1441.

[44] Lawn SD, Bangani N, Vogt MT, et al. Utility of interferon- $\gamma$ ELISPOT assay responses in highly tuberculosis-exposed patients with advanced HIV infection in South Africa. BMC Infect Dis 2007; 7: 99.

[45] Swaminathan S, Subbaraman R, Venkatesan P, et al. Tuberculin skin tests results in HIV-infected patients in India: implications for latent tuberculosis treatment. Int J Tuberc Lung Dis 2008; 12: 168173.

[46] Dheda K, Lalvani A, Miller RF, et al. Performance of a T-cellbased diagnostic test for tuberculosis infection in HIV-infected individuals is independent of CD4 cell count. AIDS 2005; 19: 20382041.

[47] Siawaya JFD, Ruhwald M, Eugen-Olsen J, Walzi G. Correlates for disease progression and prognosis of concurrent HIV/TB infection. Int J Infect Dis 2007; 11: 289-299.

[48] Goletti D, Carrara S, Vincenti D, Girardi E. T cell responses to commercial Mycobacterium tuberculosis-specific antigens in HIVinfected patients. Clin Infect Dis 2007; 45: 1652-1654.

[49] Doherty TM, Demissie A, Olobo J, et al. Immune responses to the Mycobacterium tuberculosis-specific antigen ESAT-6 signal subclinical infection among contacts of tuberculosis patients. J Clin Microbiol 2002; 4: 704-706.

[50] Andersen P, Doherty TM, Pai M, Weldingh K. The prognosis of latent tuberculosis: can disease be predicted? Trends Mol Med 2007; 13: 175-182.

[51] Goletti D, Carrara S, Vincenti D, et al. Accuracy of an immune diagnostic assay based on RD1 selected epitopes for active tuberculosis in a clinical setting: a pilot study. Clin Microbiol Infect 2006; 12: $544-550$

[52] Goletti D, Carrara S, Mayanja-Kissa H, et al. Response to $M$. tuberculosis selected RD1 peptides in Ugandan HIV-infected patients with smear positive pulmonary tuberculosis: a pilot study. BMC Infect Dis 2008; 8: 11.

[53] Hougardy JM, Schepers K, Place S, et al. Heparin-bindinghemagglutinin-induced IFN-gamma release as a diagnostic tool for latent tuberculosis. PLos ONE 2007; 2(10):e926.

[54] Elliott AM, Hurst TJ, Balyeku MN, et al. The immune response to Mycobacterium tuberculosis in HIV-infected and uninfected adults in Uganda: application of a whole blood cytokine assay in an epidemiological study. Int J Tuberc Lung Dis 1999; 3: 239-247.

[55] Fletcher HA. Correlates of immune protection from tuberculosis. Cur Mol Med 2007; 7: 319-325.

[56] Tena GN, Young DB, Eley B, et al. Failure to control growth of mycobacteria in blood from children infected with human immunodeficiency virus and its relationship to T cell function. J Infect Dis 2003; 187:1544-1551.

[57] Kampmann B, Tena-Coki GN, Nicol MP, Levin M, Eley B. Reconstitution of antimycobacterial immune responses in HIV-infected children receiving HAART. AIDS 2006; 20: 1011-1018.

[58] Badri M, Wilson D, Wood R. Effect of highly active antiretroviral therapy on incidence of tuberculosis in South Africa: a cohort study. Lancet 2002; 359: 2059-2064.

[59] Abebe F, Holm-Hansent C, Wiker HG, Bjune G. Progress in serodiagnosis of Mycobacterium infection. Scand J Immunol 2007; 66: 176-191

[60] Saltini C, Amicosante M, Girardi E, et al. Early abnormalities of the antibody response against Mycobacterium tuberculosis in human immunodeficiency virus infection. J Infect Dis 1993; 168: 1409-1414.

[61] Perkins MD, Cunnigham J. Facing the crisis: improving the diagnosis of tuberculosis in the HIV era. J Infect Dis 2007; 196(Suppl 1): $\mathrm{S} 15-27$

[62] Steingart KR, Henry M, Laal S, et al. A systematic review of commercial serological antibody detection tests for the diagnosis of extrapulmonary tuberculosis. Thorax 2007; 62: 911-918

[63] Simonney N, Chavanet P, Perronne C, et al. B-cell immune responses in HIV positive and HIV negative patients with tuberculosis evaluated with an ELISA using a glycolipid antigen. Tuberculosis $2007 ; 87: 109-122$.

[64] Amicosante M, Richeldi L, Monno L, et al. Serological markers predicting tuberculosis in human immunodeficiency virus-infected patients. Int J Tuberc Lung Dis 1997; 1: 435-440.

[65] Singh KK, Dong Y, Belisle JT, Harder J, Arora VK, Laal S. Antigens of Mycobacterium tuberculosis recognized by antibodies during incipient, sub-clinical tuberculosis. Clin Diagn Lab Immunol 2005; $12: 354-358$

[66] Elzi L, Schlegel M, Weber R, et al. Reducing tuberculosis incidence by tuberculin skin testing, preventive treatment, and antiretroviral therapy in an area of low tuberculosis transmission. Clin Infect Dis 2007; 44: 94-102.

(C) Lagrange and Herrmann; Licensee Bentham Open.

This is an open access article distributed under the terms of the Creative Commons Attribution License (http://creativecommons.org/licenses/by/2.5/), which permits unrestrictive use, distribution, and reproduction in any medium, provided the original work is properly cited. 